Robotica

http://journals.cambridge.org/ROB

Additional services for Robotica:

Email alerts: $\underline{\text { Click here }}$

Subscriptions: $\underline{\text { Click here }}$

Commercial reprints: $\underline{\text { Click here }}$

Terms of use : $\underline{\text { Click here }}$

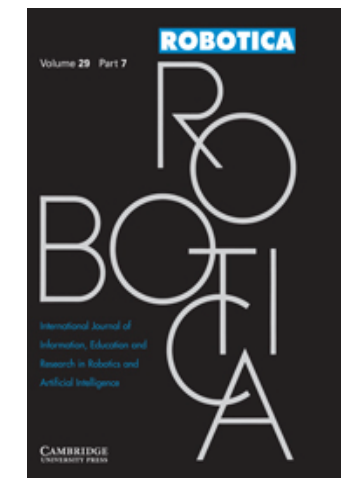

\title{
Visual servoing for path reaching with nonholonomic robots
}

Andrea Cherubini, François Chaumette and Giuseppe Oriolo

Robotica / Volume 29 / Issue 07 / December 2011, pp 1037 - 1048

DOI: 10.1017/S0263574711000221, Published online: 11 April 2011

Link to this article: http://journals.cambridge.org/abstract S0263574711000221

How to cite this article:

Andrea Cherubini, François Chaumette and Giuseppe Oriolo (2011). Visual servoing for path reaching with nonholonomic robots. Robotica, 29, pp 1037-1048 doi:10.1017/S0263574711000221

Request Permissions : $\underline{\text { Click here }}$ 


\title{
Visual servoing for path reaching with nonholonomic robots Andrea Cherubini $\dagger^{*}$, François Chaumette $†$ and Giuseppe Oriolo $\ddagger$
}

\author{
$\dagger$ INRIA Rennes - Bretagne Atlantique, Campus de Beaulieu, 35042 Rennes, France.E-mail: chaumette@irisa.fr \\ $\ddagger$ Dipartimento di Informatica e Sistemistica, Università di Roma “La Sapienza," Via Ariosto 25, 00185 Roma, Italy. \\ E-mail:oriolo@dis.uniromal.it
}

(Received in Final Form: March 9, 2011. First published online: April 11, 2011)

\begin{abstract}
SUMMARY
We present two visual servoing controllers (pose-based and image-based) enabling mobile robots with a fixed pinhole camera to reach and follow a continuous path drawn on the ground. The first contribution is the theoretical and experimental comparison between pose-based and imagebased techniques for a nonholonomic robot task. Moreover, our controllers are appropriate not only for path following, but also for path reaching, a problem that has been rarely tackled in the past. Finally, in contrast with most works, which require the path geometric model, only two path features are necessary in our image-based scheme and three in the pose-based scheme. For both controllers, a convergence analysis is carried out, and the performance is validated by simulations, and outdoor experiments on a car-like robot.
\end{abstract}

KEYWORDS: Control of robotic systems; Mobile robots; Visual servoing; Navigation; Automation.

\section{Introduction}

In recent research, automatic vehicle guidance is often done by utilizing vision sensors, ${ }^{1}$ which are very useful especially in urban environments, where numerous visual "points of interest" exist. In a city, cameras can replace or integrate GPS data ${ }^{2}$ since satellite signals can be masked by tall buildings. Various participants of the DARPA Urban Challenge (www.darpa.mil/grandchallenge) exploit vision. ${ }^{3}$ Appearance-based navigation, consisting of replaying a topological path defined by a set of images, has been accomplished in ref. [4, 5]. Apart from navigation, other mobile robot tasks that exploit camera data include localization. ${ }^{6,7}$ One of the prominent methods in visionbased navigation is visual servoing, ${ }^{8}$ which was originally developed for manipulators with a camera on their endeffector, ${ }^{9}$ but has also been applied on nonholonomic mobile robots. ${ }^{10}$ In some cases, the method relies on the geometry of the environment and on other metrical information. In this case, pose-based visual servoing is used to reduce the error, which is estimated in pose space. Other visual navigation systems use no explicit representation of the environment. In this case, image-based visual servoing techniques can be used to reduce the error, which is measured directly in the image. The image-based approach eliminates the

\footnotetext{
*Corresponding author. E-mail: acherubi@irisa.fr
}

need for image interpretation, and errors due to camera modeling and calibration. Applying such techniques on wheeled robots involves well-known problems related to the nonholonomic constraint: the linearization of these systems is uncontrollable, and smooth feedback laws stabilizing these systems do not exist.

In this paper, we focus on the path reaching task: the controller must zero some suitable error function, indicating the robot pose with respect to a path. The path is a curve drawn on the ground, and the features used for control are at the intersection of the curve with the image borders. As we will show in the nonexhaustive survey below, the path following problem ${ }^{11,12}$ has been tackled in recent research. However, for path following, the initial error is assumed small (i.e., the robot is already on the path), whereas in the path reaching problem, the initial error can be arbitrarily large. The controller in ref. [13] regulates the lateral displacement and orientation of the vehicle at a lookahead distance. Frezza et al. ${ }^{14}$ approximate the path by feasible cubic Bsplines and apply feedback linearization on the derivatives of the splines at the wheel axle. In refs. [15 and 16], straight line following is implemented, respectively, for a car-like and hexapod robot. All these controllers are posebased and require a complete geometric representation of the path. In other works, image-based techniques have been used to avoid complete knowledge of the path geometry. In ref. [17], a straight line follower for a mobile robot with para-catadioptric camera is presented. Coulaud et al. ${ }^{18}$ design a novel controller and discuss the stability of an equilibrium trajectory: for circular paths, asymptotic stability is guaranteed, whereas for continuously differentiable path curvature, the tracking error is bounded. In ref. [19], the path is approximated by a fuzzy function, which drives the steering velocity. Two-step techniques enable robot pose regulation, using a ceiling camera, in ref. [20].

We present two controllers (pose-based and image-based), enabling nonholonomic robots with a fixed pinhole camera to reach, and follow a continuous path on the ground. The development of the two schemes has been described in refs. [21 and 22]. The contribution of the present article with respect to those papers is the comparison of the two schemes. To our knowledge, a comparison between posebased and image-based visual servoing for nonholonomic robot navigation has never been carried out. To achieve this, various theoretical details, and new experimental results and simulations, not present in refs. [21 and 22], have been added here. From a theoretical viewpoint, a unique 


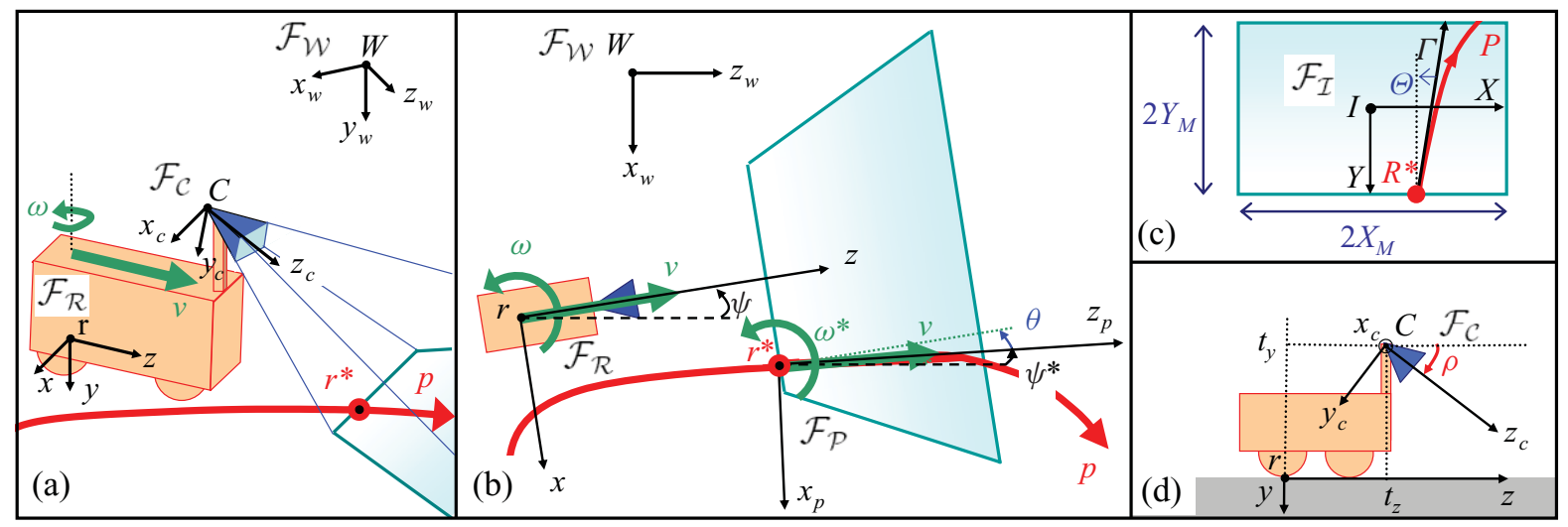

Fig. 1. (Colour Online) Relevant variables and reference frames $\mathcal{F}_{\mathcal{W}}, \mathcal{F}_{\mathcal{R}}, \mathcal{F}_{\mathcal{C}}, \mathcal{F}_{\mathcal{P}}$ and $\mathcal{F}_{\mathcal{I}}$. The task for the robot, equipped with a fixed pinhole camera is to follow the path $p$. The camera field of view and its projection on the ground are also represented. (a) Perspective view: (b) Top view: robot configuration, desired configuration, applied $(v, \omega)$ and tracking $\left(v^{*}, \omega^{*}\right)$ control variables. (c) Image plane view. (d) Side view.

representation is given for all the closed-loop systems, leading to a unique formulation of the convergence analysis. Moreover, in contrast with ref. [21 and 22], we numerically verify the closed-loop convergence of the two schemes, to get a deeper insight on their applicability and characteristics.

The contributions of our work are listed below:

(1) An image-based and a pose-based path reaching approach are compared.

(2) Under certain conditions, convergence is guaranteed even when the initial error is large. For this reason, we claim that our controllers are appropriate not only for path following, but also for path reaching; a problem that has not been tackled in the cited works, which impose constraints on the initial configuration.

(3) As opposed to most approaches, which require a geometric model of the path, in our image-based scheme, only two features (the position of a path point and the path tangent orientation at that point) are necessary.

(4) The system is validated in an outdoor environment, with varying light, in contrast with most cited papers, where tests have been carried out only indoor, where controlled light facilitates image processing.

(5) A convergence analysis is carried out.

The paper is organized as follows. In Section 2, the problem and variables are defined, along with the controllers, which are detailed in Sections 3 and 4. In Section 5, a convergence analysis is carried out. The experiments are presented in Sections 6-8.

\section{Problem Statement}

\subsection{Definitions}

We focus on the path reaching task for nonholonomic mobile robots equipped with a fixed pinhole camera. The ground is planar, and the path $p$ to be followed is a curve that is drawn on the ground. For the pose-based control scheme, we assume that the curve is twice differentiable in $I R^{2}$. For the image-based control scheme, the curve can be differentiable only once. A following direction is associated to the path (see Fig. 1). We name $r$ the point on the robot sagittal plane that should track the path. We define the frames (see Fig. 1): world frame $\mathcal{F}_{\mathcal{W}}\left(W, x_{w}, y_{w}, z_{w}\right)$, robot frame $\mathcal{F}_{\mathcal{R}}(r, x, y, z)$, and image frame $\mathcal{F}_{\mathcal{I}}(I, X, Y)$ ( $I$ is the image plane center). The image width and height are, respectively, $2 X_{M}$ and $2 Y_{M}$. The robot configuration in $\mathcal{F}_{\mathcal{W}}$ is

$$
q=\left[\begin{array}{lll}
x_{w} & z_{w} & \psi
\end{array}\right]^{\top},
$$

where $x_{w}$ and $z_{w}$ represent the Cartesian position of $r$ in $\mathcal{F}_{\mathcal{W}}$, and $\psi \in(-\pi,+\pi]$ is the positive counterclockwise orientation of $z$ with respect to $z_{w}$. The camera optical axis has a constant tilt offset $0<\rho<\frac{\pi}{2}$ with respect to the $z$-axis and the optical center $C$ is positioned in the robot sagittal plane at

$$
\left\{\begin{array}{l}
x=0 \\
y=t_{y}, \\
z=t_{z}
\end{array}\right.
$$

with $t_{y}<0$ and $t_{z} \in \mathbb{R}$. We also define the camera frame $\mathcal{F}_{\mathcal{C}}\left(C, x_{c}, y_{c}, z_{c}\right)$ (see Fig. 1d). We denote the control variables by $u=\left[\begin{array}{ll}v & \omega\end{array}\right]^{\top}$. These are the driving and steering velocities (positive counterclockwise) of the robot. Point $r$ is chosen as the center of rotation. Then, the state equation of the robot in the world frame is

$$
\dot{q}=\left[\begin{array}{c}
-\sin \psi \\
\cos \psi \\
0
\end{array}\right] v+\left[\begin{array}{l}
0 \\
0 \\
1
\end{array}\right] \omega .
$$

Although in this equation, we have utilized a unicycle robot model, our approach can be extended to other vehicles (e.g., car-like).

\subsection{Path reaching task}

We hereby define the path reaching task, by recalling the characteristics of path following. The difference is that in path reaching the initial error can be arbitrarily large. Recalling, ${ }^{12}$ the goal of path following is to drive the 
(a)

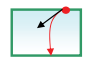

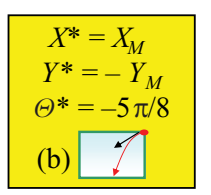

(d)

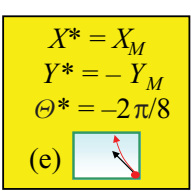

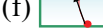

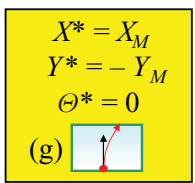

Fig. 2. (Colour Online) Outline of the control scheme, with seven possible configurations of $P$ in the image, including the three equilibrium configurations (yellow).

robot configuration $q$ to the desired configuration (shown in Fig. 1b) $q^{*}=\left[x_{w}^{*} z_{w}^{*} \psi^{*}\right]^{\top}$ such that:

- Point $r^{*}=\left[\begin{array}{lll}x_{w}^{*} & 0 & z_{w}^{*}\end{array}\right]^{\top}$ on the path $p$ defines the desired robot position.

- $\psi^{*} \in(-\pi,+\pi]$ defines the desired robot orientation, i.e., the orientation of the path tangent at $r^{*}$ in $\mathcal{F}_{\mathcal{R}}$. Note that $\psi^{*}$ is always defined, since we have assumed that the path curve can be expressed by a differentiable function.

- $u^{*}=\left[v^{*} \omega^{*}\right]^{\top}$ is the tracking control at the desired state $q^{*}$.

In practice, the goal of driving $q$ to $q^{*}$ is equivalent to zeroing the error: $e=q-q^{*} \in \mathbb{R}^{3}$. Furthermore, in path following, this goal must be achieved under two conditions:

(1) In opposition to the trajectory tracking problem,${ }^{11}$ where the desired configuration is determined by a rigid law (e.g., associated to time: $q^{*}=q^{*}(t)$ ), in path following we can arbitrarily choose the relationship that defines the evolution of $q^{*}$. Such relationship, called path following constraint, eliminates one of the three state components so that the task consists of zeroing a new 2D error $e=$ $s-s^{*} \in \mathbb{R}^{2}$, by using appropriate control inputs $v$ and $\omega$. The state dynamics is

$$
\dot{s}=\mathbf{J}(s) u=\mathbf{J}_{v}(s) v+\mathbf{J}_{\omega}(s) \omega,
$$

where $\mathbf{J}_{v}$ and $\mathbf{J}_{\omega}$ are the columns of the Jacobian $\mathbf{J}(s)$ that relates $u$ to $\dot{s}$.

(2) The robot should move at all times, while the control law ensures convergence to the path. This is the motion exigency condition defined in ref. [12]

$$
|u| \neq 0, \quad \forall s \in \mathbb{R}^{2} .
$$

In all the cited works, as well as here, motion is restricted to the forward direction $(v>0)$ for security reasons (sensing obstacles in front is easier on most robots).

\subsection{Control design}

Since in our work, the camera is the only sensor available, we want to ensure path visibility at all times. Hence, we shall use a path following constraint that keeps $r^{*}$ in the camera field of view. The path following constraint that we chose will be detailed later in the paper. Similar to refs. $[16,18$, and 19] since the only obstacle detecting sensor on our CyCab is a range scanner that points forward, we express the motion exigency as

$$
v=v^{*}=\text { const }>0,
$$

and we apply a nonlinear feedback on $\omega$ based on the features of a visible path point. Under the assumption that a portion of the path is initially visible, we utilize the features of the first (considering the path direction) visible path point $r^{*}$ of coordinates $r^{*}=\left[\begin{array}{ll}x & 0 \\ z\end{array}\right]^{\top}$ in $\mathcal{F}_{\mathcal{R}}$, which is projected to $R^{*}=$ $\left[\begin{array}{ll}X & Y\end{array}\right]^{\top}$ on the image plane (see Fig. 1c). Here, $P$ denotes the projection of the path on the image plane, $\Gamma$ denotes the oriented (according to the path direction) tangent of $P$ at $R^{*}$, and $\Theta \in(-\pi, \pi]$ denotes the angular offset from $\Gamma$ to the $-Y$-axis (positive counterclockwise). Note that $\Gamma$ and $\Theta$ are always defined since we have assumed that the path curve is differentiable in $\mathcal{F}_{\mathcal{W}}$, and this property is preserved in $\mathcal{F}_{\mathcal{I}}$.

In both control schemes that we propose (pose-based and image-based), the task is defined by the path image features. As shown in Fig. $2(\mathrm{~g})$, it consists of driving $R^{*}$ to the bottom pixel row of the image plane with vertical tangent

$$
X^{*}=0, \quad Y^{*}=Y_{M}, \quad \Theta^{*}=0 .
$$

Depending on the position of $R^{*}$ in the image, we use either of two primitive controllers: a row and a column controller. In both primitive controllers, the task is to drive the path features to a desired configuration, while $R^{*}$ is constrained to a line in the image: a row of pixels (constant $Y$ ) in the first case and a column (constant $X$ ) in the second case. These conditions determine the path following constraint introduced in Section 2.2. By using both controllers, the path can be reached from general initial configurations.

This is one of our main contributions. For example, the authors of ref. [18], which use a similar approach, assume that in the initial configuration the path already intersects the bottom pixel row, and this implies a bound on the initial position error. Instead, we simply assume that the path is visible.

Let us focus on the initial configuration where $R^{*}$ is on the top row of the image (Fig. 2a). Initially, the row controller must be used to drive $R^{*}$ to a lateral column. The column selected depends on the initial value of $\Theta$ : for $\Theta \geq 0$ (respectively, $\Theta<0$ ), the left (right) column is chosen. For the case in Fig. 2, the right column is selected. The equilibrium configuration for the top row controller (TRC) is the top right corner, with desired tangent orientation $\Theta^{*}=-5 \pi / 8 \mathrm{rad}$ (Fig. 2b). Then, the right column controller (RCC) will be used to drive $R^{*}$ (Figs. $2 \mathrm{c}$ and $2 \mathrm{~d}$ ) to the bottom right corner: $\Theta^{*}=-2 \pi / 8$ rad (Fig. 2e). Finally, the bottom row controller (BRC) will drive $R^{*}$ along the bottom row (Fig. 2f) to the center, with vertical tangent $\Theta^{*}=0$ (Fig. 2g). If the left column is initially selected, the equilibrium configurations are symmetric to the ones in the figure and they are considered reached by thresholding the point position error, i.e., the coordinates of $R^{*}$.

This composition of row and column controllers will be used in both pose-based and image-based control schemes although the state variables will be different (i.e., defined in 
the pose or the image space). In the first case, the 3D path error dynamics will depend on the curvature at the desired point, denoted $c^{*}$, and related to the tracking control inputs by

$$
c^{*}=\frac{\omega^{*}}{v^{*}} .
$$

Instead, in the image-based scheme $X, Y$, and $\Theta$ will be used, without taking into account the curvature. In both cases, by imposing the motion exigency (4), system (2) becomes

$$
\dot{s}=\mathbf{J}_{v}(s) v^{*}+\mathbf{J}_{\omega}(s) \omega,
$$

and we apply the following feedback control:

$$
\omega=-\mathbf{J}_{\omega}^{+}\left(\lambda e+\mathbf{J}_{v} v^{*}\right),
$$

with $\lambda>0$, and $\mathbf{J}_{\omega}^{+}$is the Moore-Penrose matrix pseudoinverse of $\mathbf{J}_{\omega}$. Control (7) allows $\|\omega\|$ to be minimal, under condition (3).

In practice, condition (3) is guaranteed by Eq. (4), and the term $\mathbf{J}_{v} v^{*}$ compensates the feature displacements due to the known driving velocity. In the next sections, we will instantiate this formulation for the two control schemes.

\section{Pose-Based Path Follower}

\subsection{Deriving the path $3 D$ features}

For the pose-based approach, the path 3D features in $\mathcal{F}_{\mathcal{R}}$ must be derived from the image features, by considering a pinhole camera model. The four camera parameters used for projecting are the focal length in pixels $f$, and $\rho, t_{y}$, and $t_{z}$ (see Fig. 1d). For simplicity, let us consider a normalized perspective camera model

$$
X=\frac{x}{z}, \quad Y=\frac{y}{z} .
$$

The mapping between the $\mathcal{F}_{\mathcal{I}}$ and $\mathcal{F}_{\mathcal{C}}$ coordinates of a ground point gives

$$
\begin{aligned}
x_{c} & =\frac{X t_{y}}{\sin \rho+Y \cos \rho}, \\
y_{c} & =\frac{Y t_{y}}{\sin \rho+Y \cos \rho}, \\
z_{c} & =\frac{t_{y}}{\sin \rho+Y \cos \rho} .
\end{aligned}
$$

Note that these equations do not present singularities, since by construction the image projection of any ground point has $Y>-\tan \rho$. Then, the robot frame coordinates of the ground point can then be easily derived, by using the homogeneous transformation from $\mathcal{F}_{\mathcal{C}}$ to $\mathcal{F}_{\mathcal{R}}$. For the orientation of the tangent at $r^{*}$, we obtain

$$
\begin{aligned}
\theta= & \operatorname{ATAN} 2(\sin \Theta(\sin \rho+Y \cos \rho) \\
& -X \cos \Theta \cos \rho, \cos \Theta) .
\end{aligned}
$$

To derive $c^{*}$, the path points "near" $R^{*}$ are first projected to $\mathcal{F}_{\mathcal{R}}$. Then, the equation of the path osculating circle in $r^{*}$ (thus, the value of $c^{*}$ ) is derived by least square interpolation.

\subsection{Row controller}

The goal of the pose-based row controller is to drive $(x, z, \theta)$ to a desired state $\left(x^{*}, z^{*}, \theta^{*}\right)$ while constraining $R^{*}$ to a row in the image $Y=$ const $=Y^{*}$. This is equivalent to zeroing the error $e=\left[\begin{array}{ll}x-x^{*} & \theta-\theta^{*}\end{array}\right]^{\top}$, while constraining $r^{*}$ to the projection of the row on the ground (see Fig. 1b), which is equivalent to applying the path following constraint:

$$
z=\mathrm{const}=z^{*}=\frac{t_{y}}{\sin \rho+Y^{*} \cos \rho} .
$$

This equation can be projected in the path frame $\mathcal{F}_{\mathcal{P}}$ (see Fig. 1b), where the robot coordinates are $r=\left[\begin{array}{lll}x_{p} & 0 & z_{p}\end{array}\right]^{\top}$. Frame $\mathcal{F}_{\mathcal{P}}$ lies on the path, with origin at $r^{*}, y_{p}$ parallel to $y$, and $z_{p}$ coincident with the path tangent at $r^{*}$ in the following direction. For the nonholonomic model (1), the errors dynamics in $\mathcal{F}_{\mathcal{P}}$ can be derived, as we have shown in ref. [21] to obtain

$$
\left\{\begin{array}{l}
\dot{x}_{p}=\omega^{*} z_{p}-v \sin \theta \\
\dot{z}_{p}=-v^{*}-\omega^{*} x_{p}+v \cos \theta \\
\dot{\theta}=\omega-\omega^{*}
\end{array}\right.
$$

Then, plugging Eq. (8) into $\dot{z}=\frac{\mathrm{d}}{\mathrm{d} t}\left(x_{p} \sin \theta-z_{p} \cos \theta\right)=$ 0 leads to

$$
v-v^{*} \cos \theta+\omega x=0 .
$$

This expression of the path following constraint relates the desired $\left(v^{*}\right)$ and applied $(v)$ driving robot velocities. Similarly, replacing Eqs. (8) and (9) in the expression of $\dot{x}$ yields

$$
\dot{x}=(\tan \theta) v+\left(z^{*}+x \tan \theta\right) \omega .
$$

On the other hand, replacing Eqs. (5) and (9) in the third equation of (8) leads to

$$
\dot{\theta}=\left(-\frac{c^{*}}{\cos \theta}\right) v+\left(1-\frac{c^{*} x}{\cos \theta}\right) \omega .
$$

Hence, the system state equations are

$$
\begin{aligned}
& {\left[\begin{array}{c}
\dot{x} \\
\dot{\theta}
\end{array}\right]=\mathbf{J}_{v} v+\mathbf{J}_{\omega} \omega \quad \text { with: } \mathbf{J}_{v}=\left[\begin{array}{c}
\tan \theta \\
-\frac{c^{*}}{\cos \theta}
\end{array}\right],} \\
& \mathbf{J}_{\omega}=\left[\begin{array}{c}
z^{*}+x \tan \theta \\
1-\frac{c^{*} x}{\cos \theta}
\end{array}\right],
\end{aligned}
$$

under the constraint that $|\theta| \neq \frac{\pi}{2}$, which can be avoided by temporarily using the pose-based column controller while $\Gamma$ is parallel to $X$. 


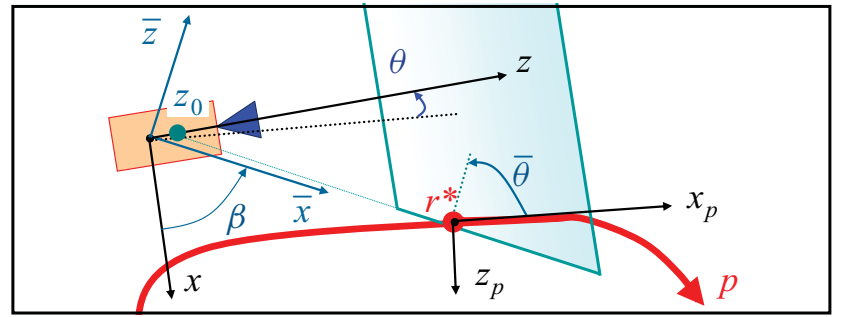

Fig. 3. (Colour Online) Relevant variables utilized for the posebased column controller: frames $\mathcal{F}_{\mathcal{R}}, \mathcal{F}_{\mathcal{P}}$, and $\overline{\mathcal{F}}_{\mathcal{R}}$; robot current; and desired configuration, $z_{0}$ and $\beta$.

By imposing the motion exigency (4), the system state equations (10) become

$$
\left[\begin{array}{c}
\dot{x} \\
\dot{\theta}
\end{array}\right]=\mathbf{J}_{v} v^{*}+\mathbf{J}_{\omega} \omega
$$

This system can be controlled using the feedback law

$$
\omega=-\mathbf{J}_{\omega}{ }^{+}\left(\lambda e+\mathbf{J}_{v} v^{*}\right) .
$$

\subsection{Column controller}

The goal of the pose-based column controller is to drive ( $x$, $z, \theta)$ to a desired state $\left(x^{*}, z^{*}, \theta^{*}\right)$ while constraining $R^{*}$ to a column in the image $X=$ const $=X^{*}$. This is equivalent to constraining $r^{*}$ to the projection of the column on the ground (see Fig. 3), i.e., to the line

$$
z=z_{0}+x \tan \beta
$$

where $z_{0}$ and $\beta \in\left(-\frac{\pi}{2}, \frac{\pi}{2}\right]$ (shown in Fig. 3) are

$$
\left\{\begin{array}{l}
z_{0}=t_{z}-t_{y} \tan \rho, \\
\beta=\operatorname{ATAN} 2(1, X \cos \rho) .
\end{array}\right.
$$

Let us redefine the variables in a new frame $\overline{\mathcal{F}}_{\mathcal{R}}(r, \bar{x}, \bar{y}, \bar{z})$, obtained by rotating $\mathcal{F}_{\mathcal{R}}$ by $\beta$ around $-y$ (Fig. 3). In $\overline{\mathcal{F}}_{\mathcal{R}}$, $\bar{\theta}=\theta+\beta$ denotes the orientation error between $z_{p}$ and $\bar{z}$, the task will consist of zeroing $e=\left[\bar{x}-\bar{x}^{*} \bar{\theta}-\bar{\theta}^{*}\right]^{\top}$, under the path following constraint:

$$
\bar{z}=\text { const }=\bar{z}^{*}
$$

$\mathbf{L}_{s}=\left[\begin{array}{ccc}-\frac{1}{z_{c}} & 0 & \frac{X}{z_{c}} \\ 0 & -\frac{1}{z_{c}} & \frac{Y}{z_{c}} \\ \frac{C \rho C^{2} \Theta}{t_{y}} & \frac{C \rho C \Theta S}{t_{y}} & -\frac{C \rho C \Theta(Y S \Theta+X C \Theta)}{t_{y}}\end{array}\right.$

with $\bar{z}^{*}=z_{0} \cos \beta$. Hence, as before, but in $\overline{\mathcal{F}}_{\mathcal{R}}$, using Eq. (8), simple calculations yield

$$
v^{*} \cos \bar{\theta}-v \cos \beta-\omega \bar{x}=0,
$$

and using Eqs. (8) and (11) gives

$$
\dot{\bar{x}}=(\tan \bar{\theta} \cos \beta-\sin \beta) v+\left(\bar{z}^{*}+\bar{x} \tan \bar{\theta}\right) \omega .
$$

On the other hand, replacing Eqs. (5) and (11) in the third equation of (8) leads to

$$
\dot{\bar{\theta}}=\left(\frac{-c^{*} \cos \beta}{\cos \bar{\theta}}\right) v+\left(1-\frac{c^{*} \bar{x}}{\cos \bar{\theta}}\right) \omega .
$$

Hence, the system state equations are

$$
\begin{aligned}
{\left[\begin{array}{c}
\dot{\bar{x}} \\
\dot{\bar{\theta}}
\end{array}\right] } & =\mathbf{J}_{v} v+\mathbf{J}_{\omega} \omega \quad \text { with: } \quad \mathbf{J}_{v}=\left[\begin{array}{c}
\tan \bar{\theta} \cos \beta-\sin \beta \\
-\frac{c^{*} \cos \beta}{\cos \bar{\theta}}
\end{array}\right] \\
\mathbf{J}_{\omega} & =\left[\begin{array}{c}
\bar{z}^{*}+\bar{x} \tan \bar{\theta} \\
1-\frac{c^{*} \bar{x}}{\cos \bar{\theta}}
\end{array}\right]
\end{aligned}
$$

under the constraint that $|\bar{\theta}| \neq \frac{\pi}{2}$, which can be avoided by temporarily using the pose-based row controller while $\Gamma$ is parallel to $Y$.

By imposing the motion exigency (4), the system state equations (12) become

$$
\left[\begin{array}{c}
\dot{\bar{x}} \\
\dot{\bar{\theta}}
\end{array}\right]=\mathbf{J}_{v} v^{*}+\mathbf{J}_{\omega} \omega
$$

This system can be controlled using the feedback law

$$
\omega=-\mathbf{J}_{\omega}{ }^{+}\left(\lambda e+\mathbf{J}_{v} v^{*}\right) .
$$

\section{Image-Based Path Follower}

Similarly to the pose-based path follower, the imagebased path follower utilizes a row and a column primitive controllers. However, in this case, the controllers are based on the reference path point image features instead of its 3D features. Let $u_{c}=\left[\begin{array}{llllll}v_{c, x} & v_{c, y} & v_{c, z} & \omega_{c, x} & \omega_{c, y} & \omega_{c, z}\end{array}\right]^{\top}$ denotes the robot velocity expressed in $\mathcal{F}_{\mathcal{C}}$. The interaction matrix $\mathbf{L}_{s}$, which relates the dynamics of visual features $s=(X, Y, \Theta)$ to $u_{c}$, has been derived, for the normalized perspective camera model, in ref. [9]:

$$
\left.\begin{array}{ccc}
X Y & -1-X^{2} & Y \\
1+Y^{2} & -X Y & -X \\
-(Y S \Theta+X C \Theta) C \Theta & -(Y S \Theta+X C \Theta) S \Theta & -1
\end{array}\right]
$$

with $C \Theta, S \Theta, C \rho$, and $S \rho$, respectively, denoting $\cos \Theta$, $\sin \Theta, \cos \rho$, and $\sin \rho$. This expression is an approximation since the observed 3D point corresponding to $R^{*}$ is varying. At low velocities, this assumption is valid, and the visual 
feature dynamics can be expressed by

$$
\dot{s}=\mathbf{L}_{s} u_{c}+\frac{\partial s}{\partial t} .
$$

The term $\frac{\partial s}{\partial t}$ corresponds to the feature motion. However, assuming low robot velocities, we can neglect this term in the control laws.

The robot velocity in $\mathcal{F}_{\mathcal{C}}$ can be expressed in function of $u=[v \omega]^{\top}$ as

$$
u_{c}={ }^{C} \mathbf{T}_{R} u,
$$

where ${ }^{C} \mathbf{T}_{R}$ is the homogeneous transformation from $\mathcal{F}_{\mathcal{R}}$ to $\mathcal{F}_{\mathcal{C}}$ :

$$
{ }^{C} \mathbf{T}_{R}=\left[\begin{array}{cc}
0 & -t_{z} \\
-\sin \rho & 0 \\
\cos \rho & 0 \\
0 & 0 \\
0 & -\cos \rho \\
0 & -\sin \rho
\end{array}\right] .
$$

In the following, we will denote by $\mathbf{T}_{v}$ and $\mathbf{T}_{\omega}$, respectively, the first and second columns of ${ }^{C} \mathbf{T}_{R}$ and by $\mathbf{L}_{X}, \mathbf{L}_{Y}$, and $\mathbf{L}_{\Theta}$ (top to bottom) the rows of $\mathbf{L}_{s}$. Replacing Eq. (15) in Eq. (14) yields

$$
\dot{s}=\mathbf{L}_{s}{ }^{C} \mathbf{T}_{R} u+\frac{\partial s}{\partial t} .
$$

This equation will be used to design the two image-based primitive controllers below.

\subsection{Row controller}

The task of the row controller is to drive $(X, \Theta)$ to a desired state $\left(X^{*}, \Theta^{*}\right)$, while constraining $R^{*}$ to a row in the image. This is equivalent to zeroing $e=\left[X-X^{*} \Theta-\Theta^{*}\right]^{\top}$, under the path following constraint:

$$
Y=\text { const }=Y^{*} \text {. }
$$

Since $\dot{Y}=0$, the system state equations are

$$
\left[\begin{array}{c}
\dot{X} \\
\dot{\Theta}
\end{array}\right]=\mathbf{J}_{v} v+\mathbf{J}_{\omega} \omega+\left[\begin{array}{c}
\frac{\partial X}{\partial t} \\
\frac{\partial \Theta}{\partial t}
\end{array}\right],
$$

where the expressions of $\mathbf{J}_{v}$ and $\mathbf{J}_{\omega}$ can be derived from Eq. (16)

$$
\mathbf{J}_{v}=\left[\begin{array}{l}
\mathbf{L}_{X} \\
\mathbf{L}_{\Theta}
\end{array}\right] \mathbf{T}_{v}, \quad \mathbf{J}_{\omega}=\left[\begin{array}{l}
\mathbf{L}_{X} \\
\mathbf{L}_{\Theta}
\end{array}\right] \mathbf{T}_{\omega} .
$$

By imposing the motion exigency (4), the system state equations (17) become

$$
\left[\begin{array}{c}
\dot{X} \\
\dot{\Theta}
\end{array}\right]=\mathbf{J}_{v} v^{*}+\mathbf{J}_{\omega} \omega+\left[\begin{array}{c}
\frac{\partial X}{\partial t} \\
\frac{\partial \Theta}{\partial t}
\end{array}\right] .
$$

This system can be controlled using the feedback law

$$
\omega=-\mathbf{J}_{\omega}{ }^{+}\left(\lambda e+\mathbf{J}_{v} v^{*}\right) .
$$

Note that, as aforementioned, this control law does not compensate the terms $\frac{\partial X}{\partial t}$ and $\frac{\partial \Theta}{\partial t}$.

\subsection{Column controller}

The task of the column controller is to drive $(Y, \Theta)$ to a desired state $\left(Y^{*}, \Theta^{*}\right)$ while constraining $R^{*}$ to a column in the image. This is equivalent to zeroing $e=\left[Y-Y^{*} \Theta-\right.$ $\left.\Theta^{*}\right]^{\top}$, under the path following constraint:

$$
X=\text { const }=X^{*} .
$$

Since $\dot{X}=0$, the system state equations are

$$
\left[\begin{array}{c}
\dot{Y} \\
\dot{\Theta}
\end{array}\right]=\mathbf{J}_{v} v+\mathbf{J}_{\omega} \omega+\left[\begin{array}{c}
\frac{\partial Y}{\partial t} \\
\frac{\partial \Theta}{\partial t}
\end{array}\right],
$$

where the expressions of $\mathbf{J}_{v}$ and $\mathbf{J}_{\omega}$ can be derived from Eq. (16)

$$
\mathbf{J}_{v}=\left[\begin{array}{l}
\mathbf{L}_{Y} \\
\mathbf{L}_{\Theta}
\end{array}\right] \mathbf{T}_{v}, \quad \mathbf{J}_{\omega}=\left[\begin{array}{l}
\mathbf{L}_{Y} \\
\mathbf{L}_{\Theta}
\end{array}\right] \mathbf{T}_{\omega} .
$$

By imposing the motion exigency (4), the system state equations (18) become

$$
\left[\begin{array}{c}
\dot{Y} \\
\dot{\Theta}
\end{array}\right]=\mathbf{J}_{v} v^{*}+\mathbf{J}_{\omega} \omega+\left[\begin{array}{c}
\frac{\partial Y}{\partial t} \\
\frac{\partial \Theta}{\partial t}
\end{array}\right] .
$$

This system can be controlled using the feedback law

$$
\omega=-\mathbf{J}_{\omega}^{+}\left(\lambda e+\mathbf{J}_{v} v^{*}\right) .
$$

As aforementioned, this control law does not compensate the terms $\frac{\partial Y}{\partial t}$ and $\frac{\partial \Theta}{\partial t}$.

\section{Convergence of the Primitive Controllers}

The four dynamic systems that we have studied can all be expressed by Eq. (6), and the corresponding primitive controllers, by Eq. (7), with the components of the 2D vectors $s, \mathbf{J}_{v}, \mathbf{J}_{\omega}$, and $e$ recalled in Table I. This general formulation will be exploited, in the following, to analyze the convergence of all four closed loop systems. A sufficient condition for convergence of control law (7) is defined in the following theorem.

Theorem 1 Under assumption

$$
e \notin \operatorname{ker} \boldsymbol{J}_{\omega}^{+},
$$


Table I. Components of $s, \mathbf{J}_{v}, \mathbf{J}_{\omega}$, and $e$ for the four controllers.

\begin{tabular}{llllll}
\hline \multirow{2}{*}{$\begin{array}{lllll}\text { Control scheme } \\
\text { Primitive controller }\end{array}$} & Row & \multicolumn{2}{c}{ Pose-based } & \multicolumn{2}{c}{ Image-based } \\
\cline { 2 - 3 }$s_{1}$ & $x$ & Column & & Row & Column \\
$s_{2}$ & $\theta$ & $\bar{\theta}$ & & $Y$ \\
$\mathbf{J}_{v 1}$ & $\tan \theta$ & $\tan \bar{\theta} \cos \beta-\sin \beta$ & & $\mathbf{L}_{X} \mathbf{T}_{v}$ & $\mathbf{L}_{Y} \mathbf{T}_{v}$ \\
$\mathbf{J}_{v 2}$ & $-c^{*} / \cos \theta$ & $-c^{*} \cos \beta / \cos \bar{\theta}$ & & $\mathbf{L}_{\Theta} \mathbf{T}_{v}$ & $\mathbf{L}_{\Theta} \mathbf{T}_{v}$ \\
$\mathbf{J}_{\omega 1}$ & $z^{*}+x \tan \theta$ & $\bar{z}^{*}+\bar{x} \tan \bar{\theta}$ & & $\mathbf{L}_{X} \mathbf{T}_{\omega}$ & $\mathbf{L}_{Y} \mathbf{T}_{\omega}$ \\
$\mathbf{J}_{\omega 2}$ & $1-c^{*} x / \cos \theta$ & $1-c^{*} \bar{x} / \cos \bar{\theta}$ & & $\mathbf{L}_{\Theta} \mathbf{T}_{\omega}$ & $\mathbf{L}_{\Theta} \mathbf{T}_{\omega}$ \\
$e_{1}$ & $x-x^{*}$ & $\bar{x}-\bar{x}^{*}$ & & $X-X^{*}$ & $Y-Y^{*}$ \\
$e_{2}$ & $\theta-\theta^{*}$ & $\bar{\theta}-\bar{\theta}^{*}$ & & $\Theta-\Theta^{*}$ & $\Theta-\Theta^{*}$ \\
\hline
\end{tabular}

a sufficient condition for the convergence of the closed loop system at the desired state $s^{*}$ is

$$
\lambda>\frac{e^{\top}\left(\boldsymbol{J}_{v}-\boldsymbol{J}_{\omega} \boldsymbol{J}_{\omega}{ }^{+} \boldsymbol{J}_{v}\right)}{e^{\top} \boldsymbol{J}_{\omega} \boldsymbol{J}_{\omega}{ }^{+} e} v^{*} .
$$

Proof: Let us consider the Lyapunov function $V(e)=\frac{e^{\top} e}{2}$, which is positive definite $\forall e \neq 0$. The time derivative of $V$ along the closed-loop system using Eqs. (6) and (7) is

$$
\dot{V}=e^{\top} \dot{s}=e^{\top}\left(\mathbf{J}_{v} v^{*}-\mathbf{J}_{\omega} \mathbf{J}_{\omega}^{+}\left(\lambda e+\mathbf{J}_{v} v^{*}\right)\right) .
$$

Since $v^{*}>0, \dot{V}$ is negative definite if and only if

$$
e^{\top}\left(\mathbf{J}_{v}-\mathbf{J}_{\omega} \mathbf{J}_{\omega}{ }^{+} \mathbf{J}_{v}\right)-\frac{\lambda}{v^{*}} e^{\top} \mathbf{J}_{\omega} \mathbf{J}_{\omega}^{+} e<0 .
$$

Since we have assumed that $\mathbf{J}_{\omega}^{+} e$ is nonnull, we also have $\mathbf{J}_{\omega}^{\top} e \neq 0$. As a consequence, $e^{\top} \mathbf{J}_{\omega} \mathbf{J}_{\omega}{ }^{+} e=\left(\mathbf{J}_{\omega}{ }^{\top} e\right)^{2} / \mathbf{J}_{\omega}{ }^{\top} \mathbf{J}_{\omega}>0$ and condition (21) can be rewritten as

$$
\frac{e^{\top}\left(\mathbf{J}_{v}-\mathbf{J}_{\omega} \mathbf{J}_{\omega}{ }^{+} \mathbf{J}_{v}\right)}{e^{\top} \mathbf{J}_{\omega} \mathbf{J}_{\omega}{ }^{+} e}<\frac{\lambda}{v^{*}},
$$

which leads to condition (20), since $\mathbf{J}_{\omega} \mathbf{J}_{\omega}{ }^{+} \neq \mathbf{I}$.

We have decided not to present the complete expressions of Eqs. (19) and (20) for the four controllers, since these were very lengthy. However, they can be derived by using the vector component values in Table I. In all four cases, Eq. (19) is an inequality constraint that is linear in $e$ and depends on both the features and the robot parameters, hence very difficult to verify analytically. Instead, we will verify it numerically in Section 6. Equation (20) gives a sufficient condition for convergence of the closed loop system at $e=0$, valid for all four primitive controllers. Note that this equation cannot be verified off line: since it is related to the state, the second part of Eq. (20) evolves during the experiment. However, Theorem 1 can be used to guarantee the convergence of the closed loop system at run-time, by updating the gain $\lambda$ at every iteration, to meet Eq. (20). Then, since, under assumption (19), all primitive controllers converge to their equilibrium point, the complete control scheme, which is defined as a cascade of the primitive controllers, will also converge. An exception occurs if the pose-based scheme singularities $\left(\theta=\frac{\pi}{2}\right.$ and $\left.\bar{\theta}=\frac{\pi}{2}\right)$ are reached. As we mentioned in Section 3 , these are avoided by switching temporarily to the other primitive controller. In this paper, however, we do not analyze the convergence of the complete system in the particular case when the switching is done. The switching was never required in the numerous experiments that we carried out.

\section{Experimental Setup}

We hereby report the experiments obtained with the two control schemes. Videos are available at www.irisa.fr/lagadic/ demo/demo-cycab-path-following/cycab-path-following.

Experiments took place outdoor using a CyCab, in varying light conditions. Our $\mathrm{CyCab}$ is a four-wheel steered intelligent vehicle equipped with a $70^{\circ}$ field of view, forward looking, B\&W Marlin F-131B camera with image resolution $320 \times 240$ pixels. It is used in car-like mode (only the front wheels are used for steering). This induces a bound on the curvature, which, for our CyCab, is $|c|<0.35 \mathrm{~m}^{-1}$. The path features are tracked with the ViSP software, ${ }^{23}$ which must be initialized at the beginning of the experiment by clicking on five path points indicating the desired path following direction. This is the only human intervention: at run-time, the tracker detects the position of $R^{*}$ and selects the corresponding primitive controller. The tracker proved always effective, and the path was never lost. In the real experiments, we set $v^{*}=0.2 \mathrm{~ms}^{-1}$. This velocity was limited for security reasons because of the low camera frequency $(10 \mathrm{~Hz})$. The system was coarsely calibrated to obtain $f=240$ pixels, $\rho=0.545 \mathrm{rad}, t_{y}=-0.55 \mathrm{~m}$, and $t_{z}=1.63 \mathrm{~m}$. Ideally, the vehicle trajectory should be assessed using a sensor independent from the camera. This was possible in the simulations, by using an ideal GPS, but since such sensor is not available on our CyCab, the camera was used to qualitatively assess the performance during the real experiments.

For each of the two control schemes, experiments with three different initial conditions have been carried out. The three experiments are enumerated below:

(1) $\mathrm{CyCab}$ is initially positioned on the path. Hence, $R^{*}$ is on the bottom image row (Fig. 4, left). The TRC is used to drive $R^{*}$ to $X^{*}=\Theta^{*}=0$.

(2) $\mathrm{CyCab}$ is initially near the path, with $R^{*}$ on the right column (Fig. 4, center). Initially, the RCC drives $R^{*}$ to 

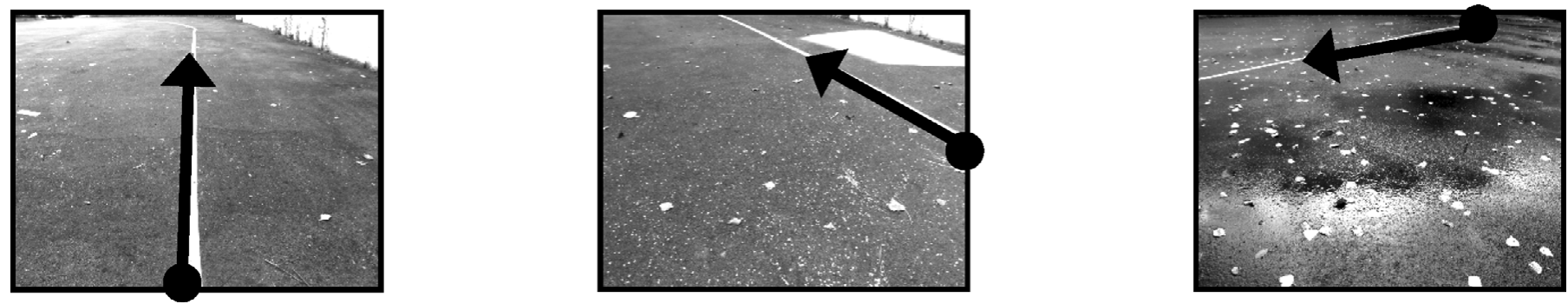

Fig. 4. Initial conditions used in the outdoor experiments. The point $R^{*}$ and tangent $\Gamma$ derived by image processing are indicated, respectively, by the black circle and arrow.

the bottom right corner. Then, the BRC drives $R^{*}$ along the bottom row to $X^{*}=\Theta^{*}=0$.

(3) $\mathrm{CyCab}$ is initially far from the path, with $R^{*}$ on the top row of the image (Fig. 4, right). Initially, the TRC drives $R^{*}$ to the right pixel column. Then, the RCC drives $R^{*}$ to the bottom right corner. Finally, the BRC drives $R^{*}$ along the bottom row to $X^{*}=\Theta^{*}=0$.

Moreover, we have numerically verified condition (19), as the state evolves. For the pose-based approach, with the CyCab parameters and state ranges, condition $e \notin \operatorname{ker} \mathbf{J}_{\omega}{ }^{+}$ is always met, except, of course, at $e=0$. This is true both for straight $\left(c^{*}=0\right)$ and curved $\left(c^{*} \neq 0\right)$ path portions. This implies that for any initial condition, and appropriate gain tuning to guarantee Eq. (20), the pose-based primitive controllers will converge to their equilibrium point, leading to convergence of the cascaded scheme. Instead, for the imagebased controller, Eq. (19) is not always met. In Fig. 5, we have represented in gray the state loci where Eq. (19) is not verified in the image-based scheme, and with a black cross the desired states for each primitive controller. In practice, as long as the state variables do not enter the gray loci, and the gain guarantees (20), the image-based primitive controllers will converge to their equilibrium point, leading to convergence of the cascaded scheme. If the state variables enter the gray loci, convergence cannot be guaranteed anymore (see Theorem 1). However, for both schemes, the states that do not meet Eq. (19) can be numerically determined off line, according to the robot parameters and state ranges, and this information can be used to select the appropriate scheme to avoid that the states enter the loci. In the following sections, the loci in Fig. 5 will be used to verify Eq. (19) during the experiments.

\section{Simulations}

For simulations, we have adopted Webots (www.cyberbotics. com), an environment for modeling and controlling mobile robots, where we have designed a robot with the same characteristics as CyCab. A circular path of radius $12.5 \mathrm{~m}$ (i.e., $c^{*}=$ const $=0.08 \mathrm{~m}^{-1}$ ) has been drawn, and the two control schemes (pose-based and image-based) have been simulated starting with the path intersecting the right column (see Fig. 4, center). The RRC and BRC are used. All gains are tuned off line to avoid abrupt changes in the steering velocity at the changing point.

With both control schemes, the robot is able to reach and follow the path, and condition (19) is always met. For the image-based case, the robot positions and processed images during the simulation are shown in Fig. 6. The relevant variables (state errors and applied curvature $c=\omega / v^{*}$ ) for the two schemes are plotted in Fig. 7. For the image-based error, instead of $e_{1}=X-X^{*}$ and $e_{1}=Y-Y^{*}$, we have plotted the scaled values $e_{1, n}=\frac{X-X^{*}}{2 X_{X}}$ for the row controller and $e_{1, n}=\frac{Y-Y^{*}}{2 Y_{M}}$ for the column controller. Note that the pose-based controller initially saturates the curvature to its maximum $0.35 \mathrm{~m}^{-1}$, to enable path reaching. Since in Webots, we can add a GPS to the robot, the controllers have been assessed by measuring the distance from the path. For the pose-based controller, the average distance is $4.1 \mathrm{~cm}$, whereas for the image-based controller, it is $4.3 \mathrm{~cm}$. Both results are excellent since they are below $0.5 \%$ of the path radius. In both cases, at the end of the first phase (after
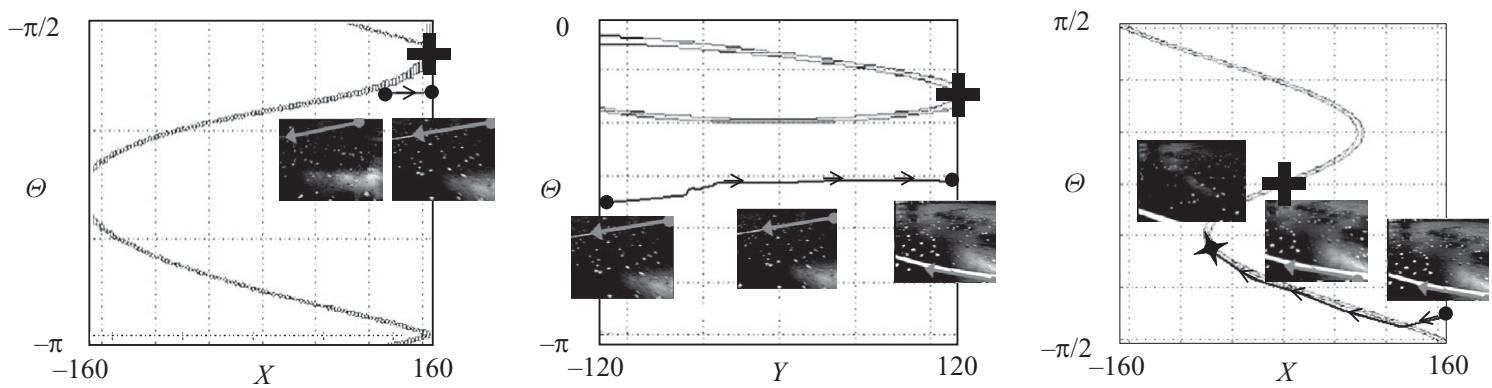

Fig. 5. For the image-based scheme, the state loci $\left(X, Y\right.$ in pixels, $\Theta$ in rad) where $e \in \operatorname{ker} \mathbf{J}_{\omega}^{+}$are shown in gray for: TRC (left), RCC (center), and BRC (right). The desired states are indicated with the black crosses. The loci (black curves) and images during the third image-based experiment are also indicated. 


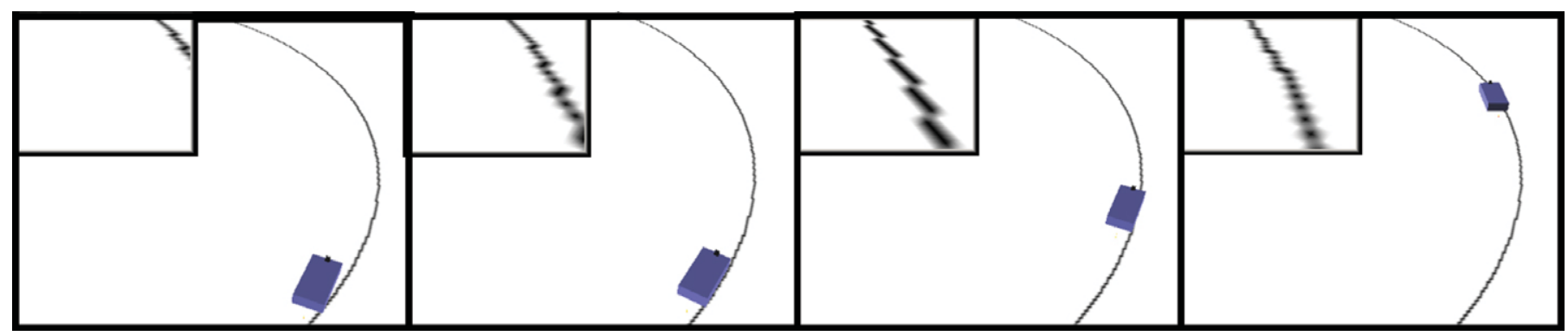

Fig. 6. (Colour Online) Image-based simulation with robot positions and corresponding images.

the RRC has been applied), the orientation error $e_{2}$ has not reached 0 because the $\mathrm{BRC}$ is activated only by $e_{1}$. Nevertheless, when the BRC is applied, the tracking errors converge, and the mean of the curvature at steady state is as expected: $c^{*}=0.08 \mathrm{~m}^{-1}$.

\section{Experiments}

After the simulations, the two control schemes have been tested outdoor on the real CyCab. The path is composed of two straight lines of length $6 \mathrm{~m}$ joined by a $60^{\circ}$ arc of circle of radius $10 \mathrm{~m}$ (i.e., $c^{*}= \pm 0.1 \mathrm{~m}^{-1}$, with the sign of $c^{*}$ depend- ing on the path direction to be followed by the robot). The primitive controller gains are the same as in the simulations. To verify the robustness of the controllers, the experiments have been repeated with a random calibration error of either $+10 \%$ or $-10 \%$ on each of the four camera parameters.

\subsection{Pose-based experiments}

In the first pose-based experiment (see Fig. 8), $R^{*}$ is on the bottom pixel row of the image plane. The row controller is used. The evolution of the relevant variables during the experiment is shown in Fig. 9. The robot successfully follows the path, and the tracking errors (solid and dotted black
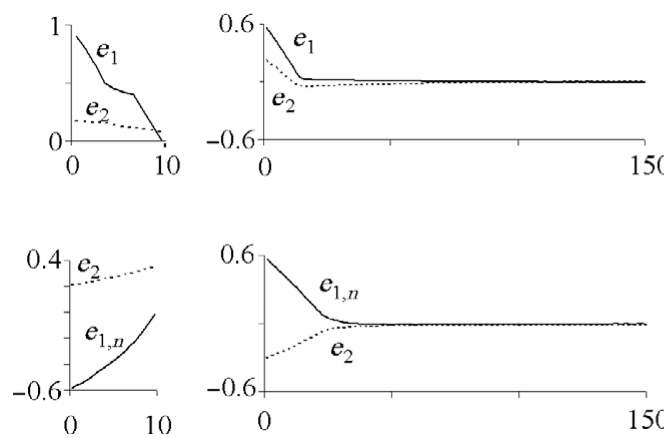
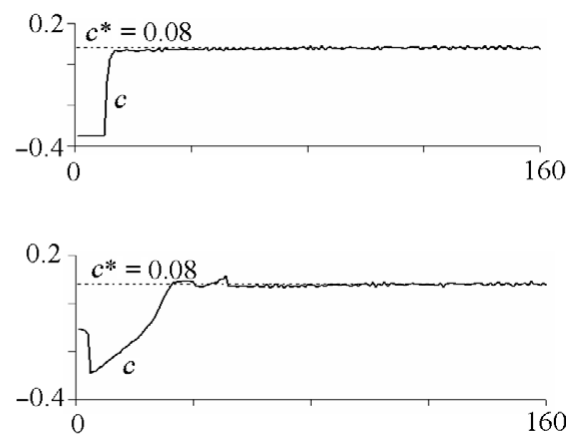

Fig. 7. Evolution of relevant variables during the pose-based (top) and image-base (bottom) simulations. Pose-based errors $e_{1}$ (solid, in $\mathrm{m})$ and $e_{2}$ (dotted, in rad), and image-based errors $e_{1, n}$ (solid, dimensionless) and $e_{2}$ (dotted, in rad) for column (left) and row (center) controllers. Applied (solid) and desired (dotted) curvatures in $\mathrm{m}^{-1}$ (right).

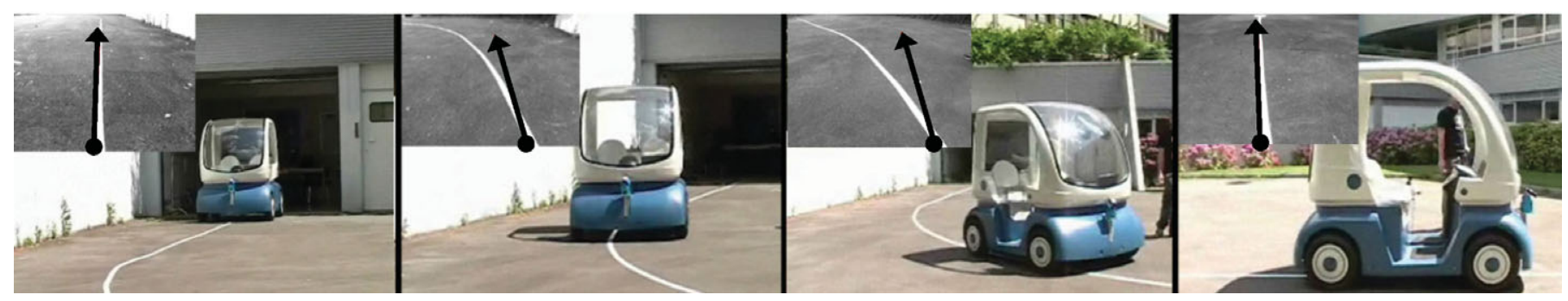

Fig. 8. (Colour Online) First pose-based experiment (CyCab is initially positioned on the path with small error), with robot positions and corresponding processed images at various iterations.
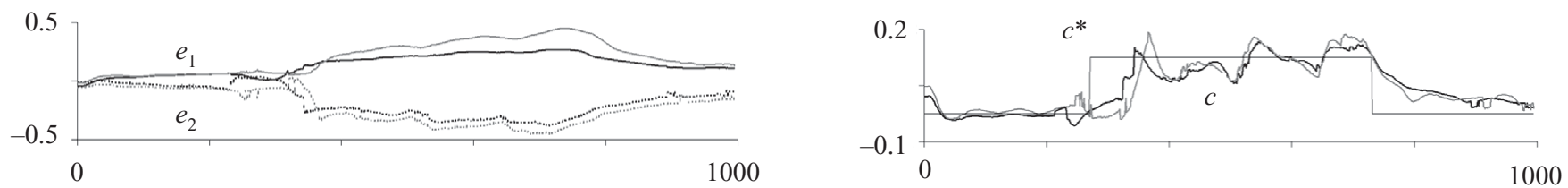

Fig. 9. Evolution of relevant variables during the first pose-based experiment, with correct (black) and coarse (gray) camera calibration. Left: errors $e_{1}$ (solid, in $\mathrm{m}$ ) and $e_{2}$ (dotted, in rad). Right: applied (thick) and desired (thin) curvatures in $\mathrm{m}^{-1}$. 

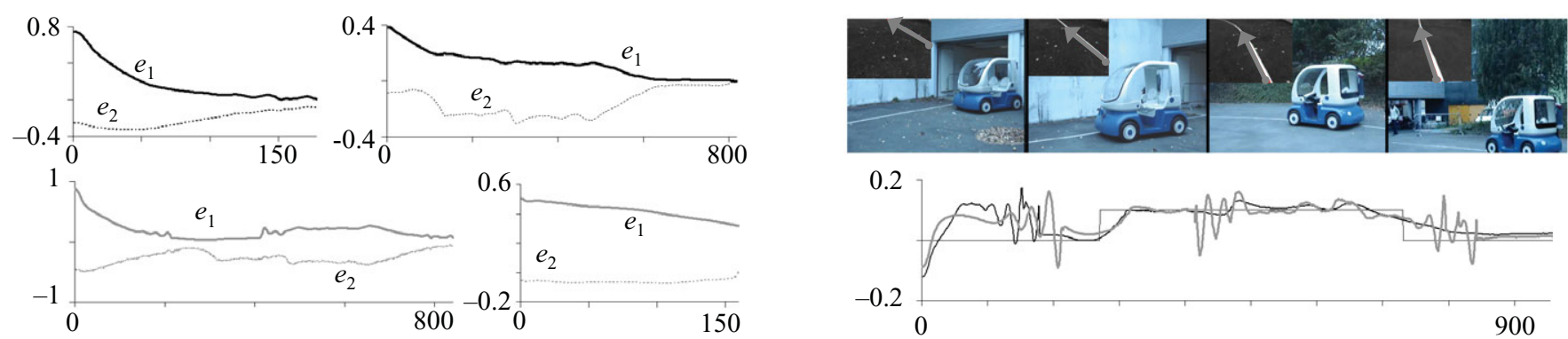

Fig. 10. (Colour Online) Variables and snapshots of the second pose-based experiment, with correct (black) and coarse (gray) calibration. Errors $e_{1}$ (solid, in $\mathrm{m}$ ) and $e_{2}$ (dotted, in rad), with RCC (left) and BRC (center), and applied (thick) and desired (thin) curvatures (right, $\left.\mathrm{m}^{-1}\right)$.

curves) are low throughout the experiment. At the end of the experiment, both errors are below 0.1. Both errors increase when the robot reaches the discontinuity in the path curvature (frame 270). Correspondingly, $\omega$ and, therefore, $c=\omega / v^{*}$ increase to compensate the error and enable $\mathrm{CyCab}$ to follow the curve.

In the second experiment, $\mathrm{CyCab}$ is initially near the path, but with $R^{*}$ on the right column. The relevant variables (state errors, as well as path and applied curvature) are plotted in Fig. 10. The robot successfully reaches and follows the path. Again, when the robot reaches the path curve (frame 285), the error increases. However, $\omega$ compensates the error and enables the robot to follow the curve and to zero both state errors.

In the third experiment (Fig. 11), CyCab is initially far from the path, with $R^{*}$ on the top row. Once again, the robot is able to successfully follow the path. The curvature is initially saturated to $0.35 \mathrm{~m}^{-1}$ to enable the robot to reach the path. At the end of the experiment, both errors are below 0.1 .

The three pose-based experiments have been repeated with camera calibration error. The variables in the coarse calibration experiments are also shown in Figs. 9-11 (gray curves), for comparison with the calibrated case (black). Although $\mathrm{CyCab}$ follows the path in all three cases, the convergence is slower than in the calibrated experiments. In particular, in the second experiment, the performance is slightly worsened (see Fig. 10, center and bottom): the RCC convergence is slower than in the calibrated case, the final error is higher $(0.35 \mathrm{~m}$ instead of $0.05 \mathrm{~m})$, and the applied curvature oscillates more.

\subsection{Image-based experiments}

In the first image-based experiment, $R^{*}$ is on the bottom row. The relevant variables are shown in Fig. 12. CyCab follows the path, and the tracking errors are low throughout the experiment (at the end, both are below 0.03). As in the pose-based experiment, both errors increase when the robot reaches the discontinuity in the path curvature, and correspondingly, $c$ increases in order to compensate for the error. Using Fig. 5, we verify that throughout the experiment, the state variables verify condition (19).

In the second experiment, $\mathrm{CyCab}$ is initially near the path, but with $R^{*}$ on the right column. The variables are plotted in Fig. 13. CyCab successfully reaches and follows the path, and at the end, both state errors are zeroed. The initial trend of $c$ is completely different from the pose-based experiment (Fig. 10, bottom). Condition (19) is always verified.

In the third experiment, $\mathrm{CyCab}$ is initially far from the path, with $R^{*}$ on the top pixel row. The experiment fails while using the BRC, as the path exits the field of view. Tests with other values of $\lambda$ are also unsuccessful. The reason is the failure of Eq. (19) during control with the BRC, at the iterations
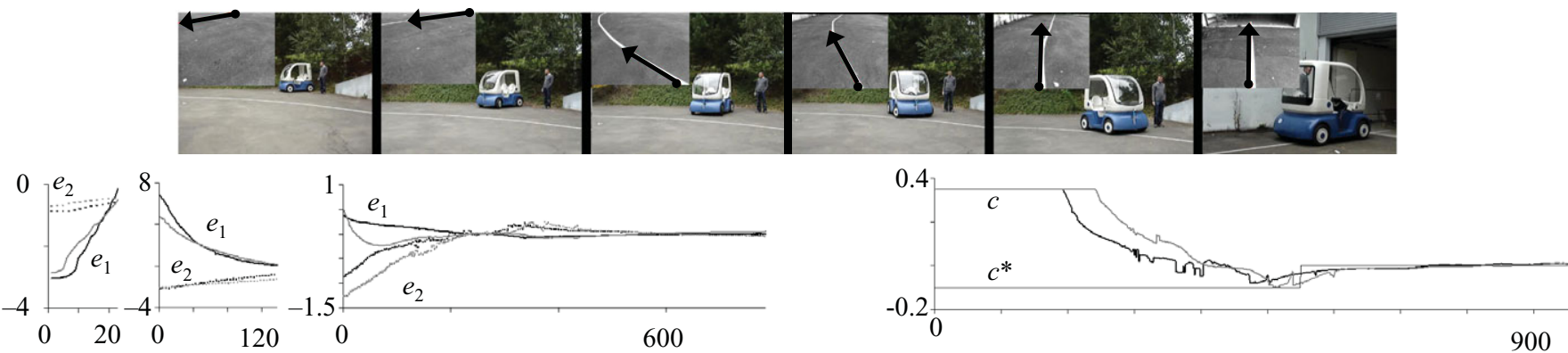

Fig. 11. (Colour Online) Snapshots and variables for the third pose-based experiment, with correct (black) and coarse (gray) calibration. Left: errors $e_{1}$ (solid, in $\mathrm{m}$ ) and $e_{2}$ (dotted, in rad), with TRC, RCC, and BRC (left to right). Right: applied (thick) and desired (thin) curvatures $\left(\mathrm{m}^{-1}\right)$.

$0.1_{1}$

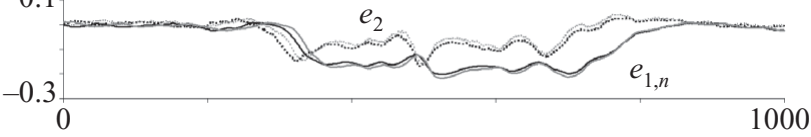

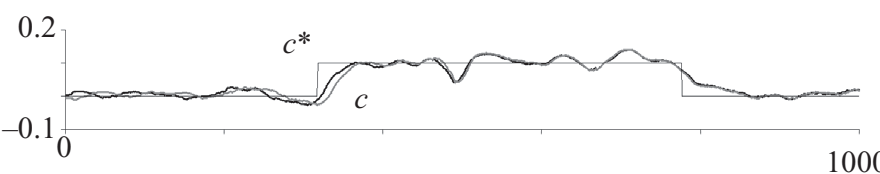

1000

Fig. 12. Evolution of relevant variables during the first image-based experiment, with correct (black) and coarse (gray) calibration. Left: errors $e_{1, n}$ (solid, dimensionless) and $e_{2}$ (dotted, in rad). Right: applied (thick) and desired (thin) curvatures in $\mathrm{m}^{-1}$. 

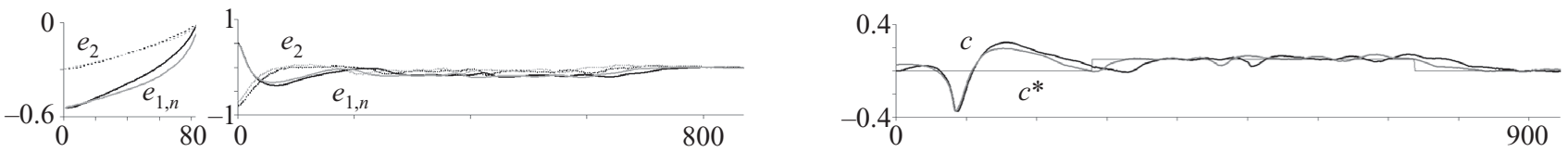

Fig. 13. Variables for the second image-based experiment, with correct (black) and coarse (gray) calibration. Errors $e_{1, n}$ (solid, dimensionless) and $e_{2}$ (dotted, in rad), with RCC (left) and BRC (center). Applied (thick) and desired (thin) curvatures (right, in $\mathrm{m}^{-1}$ ).

Table II. Error norm $|e|$ averaged over the experiments.

\begin{tabular}{lccccc}
\hline \multirow{2}{*}{$\begin{array}{l}\text { Control scheme } \\
\text { Calibration }\end{array}$} & \multicolumn{2}{c}{ Pose-based } & & \multicolumn{2}{c}{ Image-based } \\
\cline { 2 - 3 } \cline { 6 - 6 } \cline { 5 - 6 } Experiment 1 & Correct & Coarse & & Correct & Coarse \\
\hline Experiment 2 & 0.22 & 0.30 & & 0.09 & 0.09 \\
Experiment 3 & 0.21 & 0.32 & & 0.16 & 0.17 \\
& 0.38 & 0.42 & & Failed & Skipped \\
\hline
\end{tabular}

highlighted in Fig. 14. To clarify this, we have plotted in black, in Fig. 5, the state evolution during this experiment and the corresponding images. As the curves show, with TRC and RCC, the state is consistent with Eq. (19). Instead, during control with the BRC, the state error enters the kernel of $\mathbf{J}_{\omega}{ }^{+}$, and Eq. (6) cannot be controlled using Eq. (7).

As we mentioned, a flaw of the image-based scheme is that it does not consider the curvature $c^{*}$ nor the feature motion $\frac{\partial s}{\partial t}$ (see Eq. (14)). This is relevant here and causes the failure. In fact (see the third snapshot on the left of Fig. 14), the BRC is activated with a large error on $e_{2}$, in a critically curve path portion. In contrast with the pose-based scheme, the error cannot be regulated since the curvature is not in the feedback law. However, the initial state of the BRC controller cannot be changed, since it is determined by the fact that the gain $\lambda$ used with TRC and RRC must be tuned to saturate the $c$ to its maximum $0.35 \mathrm{~m}^{-1}$.

The two successful image-based experiments have been repeated by considering camera calibration error. The results are also shown in Figs. 12 and 13 (gray curves), for comparison with the calibrated experiments (black). The robot successfully follows the path in both experiments, and again, Eq. (19) is always verified. The convergence is slightly slower than in the calibrated experiments. However, in particular for the second experiment, the image-based approach outperforms the pose-based approach when the camera is coarsely calibrated.

\subsection{Comparison between the two control schemes}

To compare the two control schemes, in Table II, we show the error norms averaged over each of the real experiments. When the initial error is small (experiments 1 and 2), the image-based approach is better (smaller $|e|$, as seen in the table, and smoother $\omega$, as seen in the curves), and more robust to calibration errors. Instead, experiment 3 fails with the image-based approach and succeeds with the pose-based approach. As explained above, this is due to the failure of Eq. (19) for large error. In a series of simulations with path intersecting the top row with various values of the initial error, we have verified that when the initial $|e|$ is larger than 0.22 , the robot is not able to follow the path with the image-based control scheme. In all cases, the path is lost during BRC control, such as in experiment 3. The posebased simulations, instead, are all successful. In summary, image-based control is less effective when the initial error is large since it does not rely on the curvature, which, acting as a second derivative, fosters the prediction of the error dynamics. On the other hand, since it uses the curvature, which is typically more biased than path position and tangent orientation measurements, the pose-based controller is less smooth and less robust to calibration errors.

\section{Conclusions}

In this paper, we presented two visual servoing control schemes (pose-based and image-based) enabling nonholonomic mobile robots to reach and follow a path on the ground. The controllers require only a small set of path features: the position of a path point and the path tangent orientation at that point. For the pose-based controller, the path curvature at the point is also necessary. Although the two schemes had been introduced in refs. [21 and 22], this article presents a unique representation for all the closedloop systems used, which eases comparison and leads to a unique formulation of the convergence analysis. New experimental results, not present in refs. [21 and 22], have also been added here, along with the numerical analysis of the closed-loop convergence, which gives a deeper insight on the controllers characteristics. The main results of our comparison are that the pose-based controller can be used in general initial conditions, is appropriate for path reaching whereas the image-based controller, is more precise and robust, and should be preferred for path following (i.e., when the error is small). In future work, we aim at integrating the
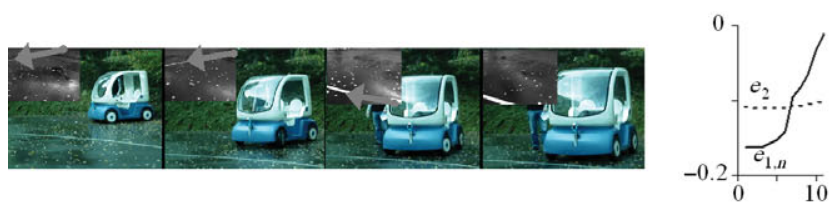
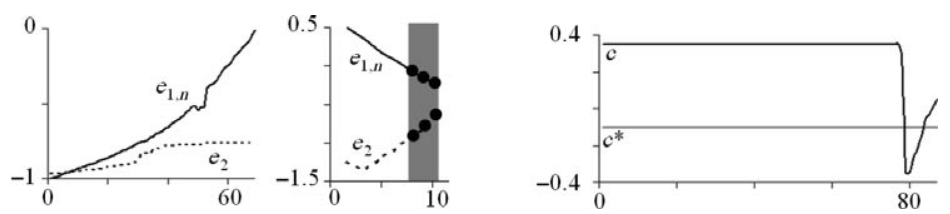

Fig. 14. (Colour Online) Snapshots and variables during the third image-based experiment (correct camera calibration). Left to right: errors $e_{1, n}$ (solid, dimensionless) and $e_{2}$ (dotted, in rad), with TRC (left), RCC (center), BRC (right), and applied (thick) and desired (thin) curvatures $\left(\mathrm{m}^{-1}\right)$. The iterations, where $e \in \operatorname{ker} \mathbf{J}_{\omega}^{+}$, are highlighted with a rectangle. 
two schemes, to design a general framework that is able to exploit the advantages of both. We also plan to vary the driving velocity $v$, in order to tackle more sophisticated scenarios.

\section{References}

1. F. Bonin-Font, A. Ortiz and G. Oliver, "Visual navigation for mobile robots: A survey," J. Intell. Robot. Syst. 53(3), 263-296 (2008).

2. M. Agrawal and K. Konolige, "Real-Time Localization in Outdoor Environments Using Stereo Vision and Inexpensive GPS," Proceedings of the International Conference on Pattern Recognition, Hong Kong (2006).

3. J. Leonard, J. How, S. Teller, M. Berger, S. Campbell, G. Fiore, L. Fletcher, E. Frazzoli, A. Huang, S. Karaman, O. Koch, Y. Kuwata, D. Moore, E. Olson, S. Peters, J. Teo, R. Truax, M. Walter, D. Barrett, A. Epstein, K. Maheloni, K. Moyer, T. Jones, R. Buckley, M. Antone, R. Galejs, S. Krishnamurthy and J. Williams "A perception-driven autonomous vehicle," J. Field Robot. 25(10), 727-774 (2008).

4. A. A. Argyros, K. E. Bekris, S. C. Orphanoudakis and L. E. Kavraki, "Robot homing by exploiting panoramic vision," Autono. Robots 19(1), 7-25 (2005).

5. E. Royer, M. Lhuillier, M. Dhome and J.-M. Lavest, "Monocular vision for mobile robot localization and autonomous navigation," Int. J. Comput. Vis. 74(3), 237-260 (2007).

6. D. Scaramuzza and R. Siegwart, "Appearance-guided monocular omnidirectional visual odometry for outdoor ground vehicles," IEEE Trans. Robot., 24(5), 1015-1026 (2008).

7. J. J. Guerrero, A. C. Murillo and C. Sagues, "Localization and matching using the planar trifocal tensor with bearing-only data," IEEE Trans. Robot. 24(2), 494-501 (2008).

8. F. Chaumette and S. Hutchinson, "Visual servo control", IEEE Robot. Autom. Mag. 13(4), 82-90 (2006) and 14(1), 109-118 (2007).

9. B. Espiau, F. Chaumette and P. Rives, "A new approach to visual servoing in robotics," IEEE Trans. Robot. Autom. 8(3), 313-326 (1992).

10. Y. Masutani, M. Mikawa, N. Maru and F. Miyazaki, "Visual servoing for non-holonomic mobile robots," Proceedings of the IEEE/RSJ/GI International Conference on Intelligent Robots and Systems, Munich, Germany (1994).

11. C. Canudas de Wit, B. Siciliano and G. Bastin, eds., Theory of Robot Control, Communication and Control Engineering (Springer-Verlag, London, 1996).
12. F. Diaz del Rio, G. Jimenez, J. L. Sevillano, S. Vicente and A. Civit Balcells, "A Generalization of Path Following for Mobile Robots," Proceedings of the IEEE International Conference on Robotics and Automation, Detroit, USA (1999).

13. M. F. Hsieh and U. Ozguner, "A Path Following Control Algorithm for Urban Driving," Proceedings of the IEEE International Conference on Vehicular Electronics and Safety, Columbus, USA (2008).

14. R. Frezza, G. Picci and S. Soatto, "A Lagrangian Formulation of Nonholonomic Path following," In: The Confluence of Vision and Control (Springer, Berlin/Heidelberg, 1998).

15. A. K. Das, R. Fierro, V. Kumar, B. Southall, B. Spletzer and J. Taylor, "Real-Time Vision-Based Control of a Nonholonomic Mobile Robot," Proceedings of the IEEE International Conference on Robotics and Automation, Seoul, Korea (2001).

16. S. Skaff, G. Kantor, D. Maiwand and A. Rizzi, "Inertial Navigation and Visual Line Following for a Dynamical Hexapod Robot," Proceedings of the IEEE/RSJ International Conference on Intelligent Robots Systems, Las Vegas, USA vol. 2, pp. 1808-1813 (2003).

17. H. H. Abdelkader, Y. Mezouar, N. Andreff and P. Martinet, "Omnidirectional Visual Servoing from Polar Lines," Proceedings of the IEEE International Conference on Robotics and Automation, Orlando, USA (2006).

18. J. B. Coulaud, G. Campion, G. Bastin and M. De Wan, "Stability analysis of a vision-based control design for an autonomous mobile robot," IEEE Trans. Robot. 22(5), 10621069 (2006).

19. A. Rezoug and M. S. Djouadi, "Visual Based Lane Following for Non-Holonomic Mobile Robot," Proceedings of the IEEE EUROCON, Saint Petersburg, Russia (2009).

20. C. Wang, W. Niu, Q. Li and J. Jia, "Visual Servoing Based Regulation of Nonholonomic Mobile Robots with Uncalibrated Monocular Camera," Proceedings of the IEEE International Conference on Control and Automation, Guangzhou, China (2007).

21. A. Cherubini, F. Chaumette and G. Oriolo, "A PositionBased Visual Servoing Scheme for Following Paths with Nonholonomic Mobile Robots," Proceedings of the IEEE/RSJ International Conference on Intelligent Robots and Systems, Nice, France (2008).

22. A. Cherubini, F. Chaumette and G. Oriolo, "An ImageBased Visual Servoing Scheme for Following Paths with Nonholonomic Mobile Robots," Proceedings of the International Conference on Control, Automation, Robotics and Vision, Hanoi, Vietnam (2008).

23. E. Marchand, F. Spindler and F. Chaumette, "ViSP for visual servoing: A generic software platform with a wide class of robot control skills," IEEE Robot. Autom. Mag. 12(4), 40-52 (2005). 